\title{
Agôn
}

Revue des arts de la scène

HS 2 | 2014

Mettre en scène le conte

\section{Un conte qui aide à vivre : conversation écrite autour du Cendrillon de Joël Pommerat}

Bérénice Hamidi-Kim et Ariane Martinez

\section{(2) OpenEdition}

Journals

Édition électronique

URL : http://journals.openedition.org/agon/3150

DOI : 10.4000/agon.3150

ISSN : 1961-8581

Éditeur

Association Agôn

Référence électronique

Bérénice Hamidi-Kim et Ariane Martinez, "Un conte qui aide à vivre : conversation écrite autour du Cendrillon de Joël Pommerat », Agôn [En ligne], HS 2 | 2014, mis en ligne le 04 mars 2015, consulté le 14 septembre 2020. URL : http://journals.openedition.org/agon/3150

Ce document a été généré automatiquement le 14 septembre 2020

Association Agôn et les auteurs des articles 


\title{
Un conte qui aide à vivre : conversation écrite autour du Cendrillon de Joël Pommerat
}

\author{
Bérénice Hamidi-Kim et Ariane Martinez
}

\section{« D'où » nous parlons... (situations)}

1 ARIANE MARTINEZ - Nous y voilà.

Nous avons convenu, il y a quelques mois, d'écrire conjointement un texte sur le Cendrillon de Pommerat. Nous avons aussi convenu que ce texte ne serait pas à proprement parler un « article » à quatre mains, forme qui lisserait nos propos et nous obligerait à accorder nos violons (avec des « et que je te corrige un mot » par-ci, et des « et que tu nuances mon propos " par là), mais bien un dialogue où nos points de vue pourraient s'expliciter, se confronter, et aussi se relayer. Nous avons choisi de converser, avec l'idée que la conversation peut aussi amener à une conversion, un infléchissement de la pensée, une nouvelle tournure aux réflexions qu'on peut se faire, quand on est seul(e), sur un spectacle. Il s'agira néanmoins d'une conversation " écrite ", où nous prendrons, chacune, le temps de développer certaines analyses.

BERENICE HAMIDI-KIM - Chère Ariane,

Une conversation, oui. Quand Marion (Boudier) m'a proposé d'écrire un texte sur la version/variation scénique du conte Cendrillon par Pommerat, je lui ai demandé s'il serait possible, plutôt que de rédiger seule un article, d'imaginer un texte à deux voix que nous écririons ensemble, parce que c'est à toi que je dois d'avoir vu ce spectacle mais aussi parce que nos discussions ont nourri ma propre réception. Autre précision, que j'ajoute ici à la demande du comité de rédaction: s'est imposé aussi d'emblée le choix de la conversation et non de l'article à quatre mains. Article: nom commun, masculin, singulier. Forme universitaire canonique dans laquelle le contexte personnel de réception est prié de bien se tenir, c'est-à-dire de se taire, alors même que l'on sait combien il informe toujours la lecture que l'on fait des œuvres. Ici, au contraire, le 
contingent débordera de toutes parts, ce sont deux femmes qui parlent, qui se connaissent depuis vingt ans, qui sont mères, amies, enseignantes-chercheuses et mille autres choses encore. Qui ne parleront pas seulement de la réception virtuelle programmée par le spectacle, comme pourrait dire l'ami Genette, mais aussi de leurs réceptions concrètes de cette œuvre, ancrées dans des corps et des psychés.

3 ARIANE MARTINEZ - ... et dans des expériences particulières, qui ont fait saillir certains aspects du conte, plutôt que d'autres. Dans mon cas, ce qui m'a frappée, c'est la dimension palliative de Cendrillon et sa résonance avec nos conceptions, nos relations contemporaines.

Notre dialogue, au sujet des spectacles de Joël Pommerat, est né en 2011, quand j'ai lu ton article consacré à Ma Chambre froide et paru, depuis, dans Friction ${ }^{1}$. Je venais, à ce moment-là, d'assister à une représentation de Cendrillon à l'Odéon. Les conditions dans lesquelles j'avais vu ce spectacle ne sont pas anodines, et c'est pourquoi je me permets de les évoquer: j'y étais en compagnie d'une «très jeune fille» de onze ans, dont le père était gravement malade, et qui est mort depuis. De cette expérience de spectatrice, j'avais gardé l'impression très forte que Cendrillon était l'une de ces pièces qui nous "aident à vivre ${ }^{2}$ ", pour reprendre la formule d'Anne-Françoise Benhamou, qui la distingue implicitement du pouvoir accordé au théâtre de "transformer le monde ». Si ces deux visions du théâtre ne s'opposent pas forcément, on voit bien ce que l'une doit à Freud (et peut-être aussi à Artaud), et ce que l'autre doit à Marx (et bien évidemment à Brecht). La force de Cendrillon me semble résider dans le fait que c'est un spectacle auquel on a envie (ou besoin) de revenir, qu'on soit enfant ou adulte, et c'est aussi au regard de cette nécessité ou de ce désir d'y revenir, que je le rapprocherai du conte. Bettelheim l'affirme :

Comme toute production artistique, le sens le plus profond du conte est différent pour chaque individu, et différent pour la même personne à certaines époques de sa vie. L'enfant saisira des significations variées du même conte selon ses intérêts et ses besoins du moment. Lorsqu'il en aura l'occasion, il reviendra au même conte quand il sera prêt à en élargir les significations déjà perçues ou à les remplacer par d'autres ${ }^{3}$.

Les contes sont des baumes au cœur qui aident à passer certains seuils. Leur fonction est autre, me semble-t-il, que l'aristotélicienne purgation des passions. Ils ne soignent pas, ils accompagnent; ils sont palliatifs, plus que thérapeutiques. Cendrillon, tel que Pommerat l'a réinventé, a joué pleinement ce rôle pour moi. C'est pourquoi, lorsque j'ai lu article sur Ma Chambre froide juste après avoir vu Cendrillon, Bérénice, et tout en partageant certaines réserves que tu formulais (bien mieux que je ne l'aurais fait) au sujet de Ma Chambre froide, je t'ai écrit pour te dire d'aller voir Cendrillon...

BERENICE HAMIDI-KIM - Oui, notre présent échange est né d'un précédent, autour de Ma Chambre froide, ou plutôt sur le billet d'humeur que j'avais écrit sur ce spectacle. Je précise l'expression car c'est bien de cela qu'il s'agissait dans ce texte, au sens où c'est une émotion forte qui m'avait fait réagir : j'avais été choquée par la teneur politique de l'œuvre, par la vision ultra pessimiste qu'elle donnait de la société actuelle, mais aussi des hommes qui la composent. Formulée sur le mode du constat, elle aboutissait à justifier un ordre social présenté comme un univers capitaliste impitoyable, inégalitaire et injuste certes, mais comme le moins mauvais système malgré tout, ou plutôt justement, tout projet de société émancipateur étant rendu caduc par la mesquinerie des individus et des groupes sociaux, dominés autant que dominants. « $\mathrm{Ce}$ monde, mauvais, ne saurait être autrement qu'il n'est", voilà ce qu'assénait ce 
spectacle, invitant subrepticement à la résignation. "Aider à vivre, transformer le monde "... Comment séparer les deux choses, Freud et Marx, psychanalyse et critique sociale? Savoir que nous avons la capacité de transformer l'existant, investiguer les profondeurs du social et de nos consciences, tenter de démêler les fils inextricables qui les unissent pour moins les subir, est-ce que cela n'aide pas à vivre, ô combien ? C'est pour ça que j'ai détesté Ma Chambre froide (mon sentiment ne s'est pas émoussé avec le temps !), parce qu'il n'aidait pas le spectateur à vivre, à mieux vivre. Et c'est pour ça que j'ai tant aimé Cendrillon : pour la vision de l'espèce humaine qui s'en dégage. Merci donc de m'avoir permis de ne pas généraliser à tort mon jugement sur cet artiste, et surtout de m'avoir fait découvrir un spectacle qui m'a bouleversée. Je parle de l'espèce humaine, bien qu'il y ait un partage évident dans la pièce entre le monde de l'enfance et celui des adultes, parce qu'à la différence d'un Bond, le changement de vision du monde d'une pièce à l'autre ne s'explique pas chez Pommerat par une conception de l'enfance comme seul âge régi par le philantropon, ce sens de l'humain cher à Aristote, avant l'inexorable déchéance de l'âme humaine dans la société corrompue des adultes. Chez Bond, les enfants sont le seul salut possible, et encore uniquement dans les pièces pour eux et centrées sur eux, en tant qu'ils sont antérieurs et extérieurs à la civilisation corruptrice qui les détruit à tout coup dans son théâtre pour adultes. Enfance, âge adulte: deux mondes séparés, dont le second dévore inexorablement la beauté innocente du premier avec ses grandes dents cyniques et médiocres. Cendrillon, autre univers, retrace le parcours qui permet à une "toute jeune fille» de devenir une femme adulte. Le passage d'un âge à l'autre est ici pensé, et qui plus est pensé comme un travail de deuil permettant une trajectoire de libération : Sandra s'allège à la fin du spectacle d'un faux-devoir, un mal-entendu qui l'empêchait de vivre, et de la culpabilité qu'il y a à survivre à sa mère. Mais le spectacle raconte aussi autre chose : le chemin pour apprendre à vivre et à goûter la vie en sachant que l'on est mortel, et que ceux qu'on aime sont mortels, en mesurant même que c'est bien là ce qui fait tout le prix de la vie, qu'elle ne soit pas éternelle, comme tente de l'expliquer à Cendrillon la fée lasse des mille et une vies qu'elle a vécues et des émotions par trop innombrables qu'elle a le vague souvenir d'avoir un jour éprouvées ${ }^{4}$.

5 ARIANE MARTINEZ - Oui, la fée n'est pas seulement celle qui évoque le bonheur des premières fois, elle est aussi celle qui met l'accent sur l'erreur, à un moment où Sandra ne sait pas encore qu'elle s'est trompée sur le sens des dernières paroles de sa mère. La fée donne une dimension comique à l'erreur, elle l'allège, elle la rend ludique, avec ses tours de cartes approximatifs : "C'est plus marrant, ça peut rater, du coup, quand je réussis, je suis folle de joie ${ }^{5} »$. Elle laisse entendre que l'erreur peut être synonyme de découverte, et qu'il faut prendre le risque d'échouer comme on court la chance.

BERENICE HAMIDI-KIM - « Une pièce qui aide à vivre ", je reviens à cette formule qui touche si juste, ici. Difficile en fait de considérer Cendrillon comme un (simple) spectacle. Il m'a plus fait l'effet d'un déclencheur d'introspection. C'est aussi que, comme toi, j'ai vu la pièce dans des circonstances personnelles particulières: alors qu'une amie très chère était gravement malade. Plus une jeune fille, mais une jeune femme, à l'aube de sa vie quand même, trop jeune pour mourir. Et morte pourtant, peu après. Cette circonstance dont l'œuvre n'était pas comptable a bien sûr affecté la perception que j'en ai eue. Mais c'est aussi que, parce qu'il suit l'itinéraire d'un personnage et encapsule une morale dans la fable, le conte incite, bien plus que d'autres formes de fiction, au retour sur soi. Faire le deuil d'une mère force une jeune 
fille à apprendre comment se construire comme femme depuis un manque si difficile à combler. Faire adulte le deuil d'une personne du même âge que soi, c'est tout autre chose. Moins impossible, mais sacrément douloureux quand même. Le manque de la personne, l'apprentissage que c'est de pouvoir s'autoriser de nouveau à penser à elle, sans être débordé par le chagrin. Mais aussi l'effet-boomerang, avec cette question en pleine face : au fait, et toi, si demain tu mourais, aurais-tu vécu ta vie? Celle que tu voulais vivre, que tu rêvais étant enfant? Cendrillon me semble ne parler que de ça. «Ma petite fille, quand je ne serai plus là il ne faudra jamais que tu cesses de penser à moi. Tant que tu penseras à moi... Je resterai en vie quelque part » : c'est ainsi que tout commence. Rappelons en deux mots la situation initiale : une très jeune fille qui refuse de vivre sa vie parce qu'elle pense que si elle oublie une seule seconde de penser à sa mère celle-ci mourra une seconde fois - ce qui n'est pas faux puisqu'elle ne vit plus que dans le souvenir de sa fille, le père voulant pour sa part « tourner la page » et « refaire [sa] vie " parce qu'il sait son temps sur terre compté et n'a pas que ça à faire, de tenter de tuer le temps en l'empêchant de s'écouler. Il sait qu'il ne peut plus s'offrir le luxe de mettre sa vie en pause. Garder vivant le souvenir de la disparue, la lourde responsabilité pèse de tout son poids sur les seules - et si frêles - épaules de Sandra. Est-ce la faute de son père ? Peut-être un peu, semble dire Pommerat - j'y reviendrai. Mais il suggère aussi que c'est avant et après tout son choix à elle. D'abord, parce que l'enfance c'est l'éternité, l'absence de conscience que le temps passe, justement, et ne reviendra pas. Mais aussi parce que "vivre est très dangereux " ${ }^{6}$, et enfin parce que se dresser contre le joug des injonctions parentales, ou plutôt de leurs ectoplasmes fantasmés, peut s'avérer une quête bien plus terrible que de terrasser quelques dragons: c'est un peu comme si Pommerat inversait la perspective de Winnicott, pointant le travail que c'est, d'apprendre à être un enfant "suffisamment bon ${ }^{7} » .$. Avant le retournement final, les mauvais traitements et tâches ménagères dégradantes qu'impose la belle-mère rencontrent à merveille la culpabilité de l'enfant : récurer la cuvette des toilettes, déboucher baignoires et lavabos, nettoyer les oiseaux morts qui s'abattent sur la maison? "Ça va me faire du bien ", "c'est dégoûtant ». De même se réjouit-elle à la vue de sa chambre-cachot : "c'est moche, ça m'correspond ». "Ce qu'on ne peut pas dire, on ne peut pas le taire ${ }^{8}$ ", le symptôme jaillit de toutes parts, par la parole et par les actes.

7 ARIANE MARTINEZ - Oui, cette expression de "déclencheur d'introspection " me semble d'autant plus vraie qu'elle rend compte, du point de vue du spectateur, d'une particularité qui ne m'avait pas sautée aux yeux quand j'avais vu le spectacle au théâtre, et qui s'avère bien plus évidente dans le film tiré de la pièce, par le jeu des plans où les visages apparaissent clairement ${ }^{9}$. Le fait que, hormis Catherine Mestoussis (la belle-mère) et Deborah Rouach (Sandra), tous les acteurs jouent plusieurs rôles ${ }^{10}$, renforce l'impression que tout le conte se déroule à l'intérieur d'une conscience (celle de Sandra devenue âgée? Celle de la fée ?). On a l'impression, comme dans un monodrame ou dans un rêve, que cette conscience se plait à réagencer les rôles, et même à produire des êtres composites, tantôt persécuteurs, tantôt complices. 


\section{Un parcours initiatique entre pensée logique et pensée magique}

8 ARIANE MARTINEZ - Parce que Ma Chambre froide est une réécriture partielle de La Bonne Âme du Se-Tchouan, tu avais, dans ton article, placé Pommerat dans la filiation de Brecht, pour montrer combien il dérogeait finalement à cette filiation. Depuis, d'autres critiques t'ont suivie dans ce sens: dans son livre sur Cendrillon, Christophe Triau évoque le désir de Pommerat de restituer, grâce au théâtre " l'étrangeté des choses ${ }^{11}$ " que l'habitude nous a fait perdre. Il rapproche cette idée de « l'étrangéification » (autre traduction possible du terme de «distanciation" en français, puisque verfremdungseffekt signifie « effet d'étrangeté »). Mais Triau finit par souligner combien Pommerat s'éloigne des moyens du théâtre épique et critique, et met en relation certains des effets de son théâtre avec l'inquiétante étrangeté freudienne ${ }^{12}$.

BERENICE HAMIDI-KIM - C'est vrai, la référence à Brecht permet de dire ce que Pommerat reprend à l'épique : au-delà du travail de réécriture de pièces de Brecht, le recyclage de certains outils, dont l'étrangéification, permise par exemple par le découplage entre le vu et l'entendu pour les spectateurs, entre le fait et le parlé pour les acteurs, dans Les Marchands. Elle permet aussi de dire l'écart fondamental, en termes de projet politique, entre La Bonne Ame et Ma Chambre froide par exemple. Mais laissonslà Brecht peut-être, car il n'aide pas beaucoup à penser Cendrillon.

10 ARIANE MARTINEZ - De mon côté, s'il fallait situer Pommerat dans une filiation, j'aurais tendance à le placer plus volontiers dans celle d'Artaud, que dans celle de Brecht bien entendu, pas dans l'idée d'un théatre de la cruauté, mais dans son rapport au spectacle, qui engage aussi un rapport au monde. En effet, Pommerat est très éloigné de la démarche critique de Brecht. S'il emprunte une chose à Brecht, c'est peut-être simplement un procédé, à savoir son utilisation des classiques ${ }^{13}$, sa manière de reprendre des situations du répertoire, pour les réinventer et les actualiser. Mais un procédé n'est pas une démarche. Si j'engage ce parallèle possible entre Pommerat et Artaud, c'est d'abord parce que, dans la revendication de Pommerat d'écrire des spectacles ${ }^{14}$ plutôt que des pièces, dans sa pratique qui consiste à "positionner la parole en rapport avec l'espace, le son et la lumière ${ }^{15}$ ", on retrouve me semble-t-il l'idée d'Artaud d'une "pièce faite de la scène directement, [...] où les délimitations habituelles des sentiments et des mots soient abandonnés ${ }^{16}$ ». Les "zones volontairement imprécises ${ }^{17}$ » de l'écriture de Pommerat, sa recherche d'un "équilibre de la lumière entre montrer et cacher, désir de voir et empêchement ${ }^{18}$ ", sa volonté de cultiver une relation "floue, ambiguë [...] avec le monde qui nous entoure ${ }^{19}$ ", m'évoquent irrésistiblement le postulat d'Artaud selon lequel « les idées claires sont, au théatre comme partout ailleurs, des idées mortes et terminée ${ }^{20} »$. Et jusque dans la manière qu'a Pommerat d'assumer la contradiction, et non de vouloir la penser et la dépasser, il me semble qu'il y a une posture très similaire à celle d'Artaud, qui rejetait le cartésianisme et empruntait volontiers à l'inconscient sa capacité à faire coexister des propositions contradictoires :

La complexité ne me fait pas peur, la contradiction non plus, l'assemblage des contraires m'attire. Nous sommes composés de contradictions. En priver le regard, c'est lui dissimuler ce qui fait la beauté de chaque chose dans ce monde. «Louis Brouillard ", le nom de ma compagnie vient de ces convictions. J'ai pensé au terme brouillard en opposition avec « clarté ", avec «l'esprit français », ce fameux dogme $\mathrm{du}$ «ce qui se pense bien s'énonce clairement». Sous-entendu, tout ce qui ne se 
maîtrise pas par la parole, n'a ni intérêt ni réalité. Autrement dit, la parole doit tout cerner. Mais, surtout, il n'y a rien d'autre d'intéressant et d'existant que ce que je peux nommer. Ça démontre un fantasme de domination par le mot, par le texte. Sans doute quelque chose qui a voulu historiquement s'opposer à une domination tyrannique, du religieux et de l'irrationnel ${ }^{21}$.

La contradiction est un moteur dramaturgique pour Pommerat. En général, dans ses pièces, il se refuse à la résoudre, comme en témoignent les deux fins incompatibles et pourtant coexistentes d'Une seule Main. Il aime à cultiver l'ambiguïté, travailler ensemble la transparence et l'opacité, laisser en suspens les positions contradictoires, comme nos rêves parviennent à le faire.

Or, Cendrillon fait bande à part dans la constellation de ses drames ambigus. C'est peutêtre cela qui fait que la pièce suscite autant l'adhésion. Elle réconcilie les adeptes de la pensée logique et ceux de la pensée magique. Pensée logique, parce qu'il y a une vraie progression dans l'histoire, progression qui permet de résoudre la contradiction incarnée au départ par Sandra (qui s'applique à survivre à sa mère pour maintenir son souvenir, mais qui se punit de lui survivre dans un même mouvement). Cette progression dramatique est dialectique et accompagne les raisonnements de Sandra : au début, la très jeune fille se fait souffrir en substituant le masochisme au deuil ; puis elle prend conscience du travail nécessaire du deuil en découvrant la situation du Prince; enfin elle fait retour sur sa propre expérience et la dépasse. Mais ce " pas à pas » du conte, qui procède par étapes claires et repérables, se fait au sein d'un monde qui, sans être merveilleux, est habité par la pensée magique, et tout d'abord la sienne, puisqu'elle est persuadée qu'il suffit de penser à sa mère morte pour qu'elle ne meure pas «en vrai ». Dans ce monde, les signes sont posés comme flous, incertains, dès le prologue, qu'il s'agisse des signes gestuels sémaphoriques faits par l'homme, ou des signes verbaux dénoncés d'entrée de jeu comme potentiellement trompeurs par la narratrice. Ce rapport ambigu au signe se prolonge dans l'intrigue, que ce soit sur un mode tragique, dans la scène des dernières paroles de la mère, ou sur un mode comique, quand la très jeune fille tente d'aiguiller la fée sur la carte qu'elle a tirée, en lui mimant, tant bien que mal, le quatre de carreau (I, 13). Il y a dans la pièce une forme d'animisme des signes: ils agitent les corps, mais demeurent obscurs, difficiles à déchiffrer. Le quiproquo est permanent, comme dans la scène 5 de la première partie, où la fille et le père se tiennent, indécis et désorientés, au seuil de la maison en verre, en dépit des indications gestuelles de la belle-mère. Sandra est perdue dans les signes, et son surnom "Cendrier ", est aussi issu du brouillard de fumée dans lequel elle est plongée par son père, qui l'enfume en faisant d'elle sa confidente au lieu d'essayer de l'aider à dépasser sa douleur. D'ailleurs, le père aussi fait «signe " à Sandra de taire le souvenir de la mère, face aux injonctions punitives de la belle-mère, signes dont on ne sait si elle les mésinterprète, ou si elle les ignore délibérément, dans son entêtement masochiste (I, 10). On peut même se demander si l'obsession ménagère de Sandra, audelà de son auto-flagellation affichée, ne reflète pas chez elle un besoin de nettoyer le monde (et sa propre conscience) de tous ces signes obscurs qui l'entachent, et dont elle ne parvient plus à saisir le sens, car elle insiste bien sur le fait qu'elle va "aimer ça ", que «ça va [lui] faire du bien » (et pas seulement lui faire les pieds). La fée ne semble pas, dans un premier temps, pouvoir aider Sandra à frayer son chemin dans cette forêt de signes, puisqu'elle rajoute de la fumée à celle du père, et l'enferme dans une boîte de magie amateur, plus sombre encore que la cave dans laquelle l'a terrée la belle-mère. Et pourtant, l'embrouillamini mental de Sandra se dissipe in extremis, grâce à la discussion avec le prince qui l'éclaire sur ses propres comportements (II, 12), et au 
retour en arrière dans la chambre de la mourante, qui éclaire son passé sous un autre jour (II, 14). Contrairement à ce qui se passe dans ce que j'appellerais les pièces-puzzle pour adultes, qui sont faites de morceaux de situations non-élucidées (Je tremble, Cercles/Fictions, La Réunification des deux Corées), l'ambiguïté n'est pas maintenue. Elle se dissout en même temps que l'intrigue se résout, et le quiproquo initial est explicité, se réduisant à une simple erreur de parcours, comme le souligne la narratrice dans les dernières paroles de la pièce : « Même les erreurs ont une fin heureusement. Alors moi je m'en vais et je me tais ${ }^{22} »$.

\section{Complexité des figures parentales}

BERENICE HAMIDI-KIM - C'est un point important il me semble à souligner chez Pommerat : si sa pâte esthétique est stable, plus que celle d'autres metteurs en scène, sa pâte politique change de consistance d'une pièce à l'autre. Comme si, animé d'un doute sur l'étoffe morale dont nous sommes faits, il la testait, au risque de faire parfois de ses personnages des cobayes. C'est aussi ce que j'ai aimé dans Cendrillon, par contraste avec d'autres spectacles: qu'il ne traite pas tous ses personnages en entomologiste uniquement dans la distance, neutralité cruelle du savant, cruauté prétendument neutre du faux cynique - mais se mette à leurs côtés. Et c'est en cela qu'il joue, jongle pourrait-on dire presque, avec les codes du conte (pas en cela seulement - témoins les scènes dansées ouvrant et clôturant le spectacle, tenant lieu du célèbre «Il était une fois » enluminé et du non moins célèbre «Ils vécurent heureux et eurent beaucoup d'enfants" - mais en cela notamment). Même s'il focalise son attention sur le personnage principal de Cendrillon, il fait cas des autres, leur conférant à tous une complexité psychologique absente, c'est le moins qu'on puisse dire, de la version originale, soumise à un schéma actantiel dont la simplicité, si elle a permis à Greimas un beau succès, a pour prix un certain simplisme des caractères. Ainsi de la belle-mère, marâtre dans toute sa splendeur, personnage certes abyssal - et abyssalement drôle de méchanceté et d'égoïsme, destructrice à l'égard de ses filles au moins autant que de sa belle-fille d'ailleurs, quand elle leur jette à la figure que « c'est à cause [d'elles] que tout est foutu ", mais pour autant émouvante et par instants bouleversante en femme, mère et épouse rongée par la frustration, par exemple quand elle s'écrie qu'elle « sen[t] depuis qu['elle est] toute petite qu['elle n'est] pas considérée à [sa] juste valeur » et espère encore, envers et contre toute la médiocrité de sa vie " qu'il va se passer quelque chose de vrai » et de grand. Complexité de la belle-mère, donc. Du père aussi, ou plutôt des pères. Survivants de leurs épouses, le père de Cendrillon et celui du prince sont des hommes bons. Mais le premier manque à sa mission de protection de son enfant, qu'il laisse à la merci de sa nouvelle femme autant que du sentiment de culpabilité d'avoir survécu à sa mère. Et le second, par faiblesse, ne parvient pas à tenir à son fils une parole de vérité, à lui dire que sa mère est morte, le laissant croire que d'interminables grèves de transport l'empêchent depuis des années de rentrer de voyage. Ni opposants, ni adjuvants, ces pères font ce qu'ils peuvent, c'est-à-dire pas beaucoup, mais un peu quand même.

ARIANE MARTINEZ - Les trois parents incarnent des attitudes éducatives différentes, voire opposées, et c'est ce que Sandra révèle indirectement quand elle qualifie de " méthode » l'incapacité du roi à annoncer à son fils que la reine est morte: « Moi ce que j'en pense de cette méthode ${ }^{23} »$. La belle-mère phallique prétend élever les enfants 
à la dure mais pour leur bien : c'est toujours « pour son bien » qu'elle persécute Sandra. Elle incarne le bon sens autoritariste dans toute sa splendeur : «Ta fille, si tu la laisses te commander, c'est elle qui va te donner des ordres ${ }^{24} »$. Et comme souvent en matière d'éducation, les principes de la belle-mère et ses actes ne coïncident pas, notamment lorsque qu'elle affiche la volonté d'une répartition «juste et équitable » des tâches, "évidemment». Le père, qui passe aux yeux de la belle-mère pour un dangereux laxiste, tente d'établir une forme de complicité avec sa fille, complicité dont les limites semblent assez délicates à poser, puisque Sandra a même accès à une photo « cochonne, "olé! olé" comme on dit entre mon père et ma mère ${ }^{25}$ ». Son père la prend régulièrement pour confidente, mais il l'abandonne à son sort, en privilégiant sa nouvelle vie avec la belle-mère ${ }^{26}$. Il est toujours sur le départ, la laissant s'installer seule dans la cave (I, 6), fuyant avec la meute quand elle s'éclabousse de merde (I, 12, dans le film, on entend même Sandra laisser échapper un vain «Papa »), ou l'abandonnant le soir du bal après avoir formulé quelques scrupules (II, 4). En cela, son rapport à sa fille résonne bien au-delà de la situation du deuil (« malheur qui heureusement n'arrive que très rarement aux enfants $\left.\aleph^{27}\right)$. Son désir de refaire sa vie très vite sans regarder derrière, ses maladroites tentatives de rapprochement font écho à un sentiment d'abandon bien plus couramment vécu aujourd'hui par les enfants des familles recomposées. Enfin, le troisième parent, le roi, surprotège son fils au point de lui cacher dix ans la mort de sa mère; il l'accompagne dans tous ses faits et gestes, même ceux que les adolescents ne devraient accomplir que seuls (la rencontre amoureuse). Autoritarisme, abandon, surprotection et copinage... Le tableau n'est pas très reluisant, mais il reflète assez bien les deux pôles extrêmes auxquels nous renvoient systématiquement les débats sur l'éducation depuis 1968, entre le Charybde des règles imposées, et le Scylla de la proximité fusionnelle. Libre à chacun de s'y reconnaître, avec tendresse, ironie, ou angoisse. C'est aussi au regard de cette actualisation des rapports parents-enfants que la pièce fait mouche.

BERENICE HAMIDI-KIM - Entourés par un amour peu sécurisant, c'est aux presque orphelins, aux mal-aimés - trop ou trop peu, trop près ou trop loin - d'être courageux. Cette incompétence des parents est aussi paradoxalement ce qui donne aux enfants la possibilité d'affirmer leurs choix. Et d'apprendre à vivre leur vie au lieu de se la faire voler par le fantôme des vies de ceux qui sont venus avant eux. La référence à $L a$ Mouette, tout sauf ornementale, sonne ainsi comme un avertissement adressé au spectateur autant qu'au personnage: derrière la marâtre acariâtre rôde l'ombre de Macha, "en deuil de la vie ", aboutissement à craindre pour la trajectoire qu'amorce Sandra au début de la pièce, sauf si...

\section{Du parcours de la très jeune fille : masochisme et empowerment}

BERENICE HAMIDI-KIM - La complexité des personnages, c'est aussi et surtout, bien sûr, celle de ce personnage-là, non pas une anti-héroïne, car elle agit, à mille lieux de la falote version originale du conte. Mais une héroïne déglinguée, renfrognée, princesse pas charmante du tout. Pommerat déplace complètement la figure de l'héroïne/victime passive qui n'a pour elle que sa fraiche beauté et son exaspérante bonté, avec constance soumise aux désirs des autres, qu'ils lui veuillent du mal (la marâtre) ou du bien (le prince). Certes, le monde extérieur est hostile, au début de l'histoire, mais sa belle- 
famille la maltraite bien moins qu'elle ne se maltraite elle-même - utilisant d'ailleurs belle-mère et demi-sœurs comme des adjuvants dans sa quête d'auto-punition. Certes, à la fin, elle rencontre un prince et ils tombent amoureux, mais c'est elle qui le sauve et ce faisant se sauve... Et si cette rencontre la façonne indéniablement, elle ne la révèle pas à elle-même. Ils s'aimeront, mais ne resteront pas ensemble, elle vivra d'autres aventures. Une femme n'a pas besoin d'un homme pour tenir debout, ce qui n'empêche pas l'amour, tout au contraire, nous dit le spectacle. Le Cendrillon de Pommerat s'inscrit ainsi dans une tendance contemporaine dont, en tant que femme et mère d'une toute petite fille, je ne peux que me réjouir : un aggiornamento idéologique des contes pour fillettes, dont participe aussi, il faut le reconnaître, le dernier succès planétaire de Disney, La Reine des neiges. Si ce dessin animé a pu faire l'objet de critiques féministes ${ }^{28}$, il n'en reste pas moins que sa morale est à mille lieux des manuels d'éducation pour jeunes filles de l'époque de nos grands-mères - et qui refleurissent hélas ici ou là, à mille lieux donc de La Belle au bois dormant, de Blanche Neige ou de Cendrillon: ce qui sauve Anna, transformée en statue de glace, ce n'est pas le baiser du prince Hans lequel s'est révélé être pour le moins décevant, arriviste criminel, sans cœur et sans panache aucun - c'est " un geste de véritable amour » accompli par... elle-même, envers sa sœur Elsa, précisément en s'interposant entre celle-ci et le prince qui voulait la tuer. Comme quoi, même les héroïnes Disney sont capables d'un peu d'empowerment, in the end... Etre le sujet et non l'objet de l'acte d'amour, et que cet amour soit philia et non eros - qui plus est un amour sororal, et donc féminin, voilà qui n'est pas banal au pays de Mickey.

ARIANE MARTINEZ - Oui, mais là où le parcours de Sandra m'intéresse beaucoup plus que celui d'Anna, c'est précisément dans l'accent mis sur le masochisme, question morale et psychologique, qui rejoint aussi une question sociale, celle de l'esclavage domestique comme cercle vicieux dont on peine à se tirer. Anna-la-princesse-cadette de La Reine des Neiges ne se remet jamais en question (y compris quand elle fait prendre des risques inconsidérés au prolétaire livreur de glace qui l'accompagne) et c'est cette assurance qui la rend insupportable à mes yeux. Elle aura toujours de quoi payer pour réparer ses gaffes, alors que Sandra-la-souillon n'en finit pas de payer de sa personne. Il lui faudra deux rencontres merveilleuses, imprévues dans l'ordre naturel et social (la fée et le prince), pour se sortir de l'exploitation de la femme par la femme, que lui ont infligée sa belle-mère et ses demi-sœurs aux grandes oreilles de loup (II, 3). L'environnement dans lequel baigne Sandra renforce sa prédisposition masochiste, liée au deuil, en y ajoutant celui des tâches ménagères, initié et alimenté par l'entourage féminin, mais aussi celui du "souffrir pour être belle», car c'est encore "pour son bien ${ }^{29}$ " que la belle-mère lui impose le corset qui lui permettra de «se tenir » (terme, bien entendu, ambivalent, puisqu'il s'agit aussi de la tenir). Le fait que ce masochisme soit porté par une jeune fille ne me semble pas anodin. Je sais bien qu'il est lié aux circonstances du deuil, et à la pression de sa famille adoptive, plus qu'à ce qu'on pourrait nommer une condition féminine. Il n'empêche qu'à situation de deuil égale, la différence de traitement avec le prince (qui s'est donné la peine de naître garçon et souverain) est notable. Lui, on le ménage, on le tient "à l'écart du monde ${ }^{30}$ " pour le préserver; elle, elle fait le ménage et plonge les mains dedans. Il n'arrive pas à grandir (en témoignent ses pantalons un peu trop longs), elle grandit trop vite (et sa petite robe blanche du début de la pièce est trop courte aux manches et aux jambes). Aussi incongru ou anachronique que cela puisse paraitre, je n'ai pu m'empêcher, la première fois que j'ai vu Cendrillon, de repenser au passage du Deuxième sexe, où Simone de 
Beauvoir analyse les pulsions masochistes des jeunes filles, en lien avec leur absences de possibilités de s'accomplir socialement :

Masochiste, puisque dans ses conduites elle accueille la douleur, elle est surtout sadique: en tant que sujet autonome, elle fouaille, bafoue, torture cette chair dépendante, cette chair condamnée à la soumission qu'elle déteste sans vouloir cependant s'en distinguer. [...] Même ses explosions de violence s'enlèvent sur un fond de résignation. Quand un jeune garçon est en révolte contre son père, contre le monde, il se livre à des violences efficaces ; il cherche querelle à un camarade, il se bat, il s'affirme à coups de poing comme sujet: il s'impose au monde, il le dépasse. Mais s'affirmer, s'imposer est interdit à l'adolescente, et c'est bien là ce qui met dans son cœur tant de révolte: elle n'espère ni changer le monde, ni en émerger ; elle se sait ou du moins se croit, et peut-être même se veut, ligotée [... $]^{31}$

Certes, dans Cendrillon, le jeune prince, lui non plus, ne s'accomplit pas dans la révolte contre un monde dont il ignore tout; mais Sandra, qui avait au départ résisté aux ordres de la belle-mère lui intimant l'ordre de poser son sac (I, 5), finit par s'enferrer dans une serviabilité excessive qui confine à l'hybris (en délivrant ses demi-sœurs des rares tâches qui leur incombent, en s'obstinant à réparer les toilettes alors que personne ne le lui demande). Si bien que sa destinée professionnelle semble tracée d'avance : servir les autres, comme elle a servi les membres de sa famille: «En plus, cette fille, c'est pas un cadeau, à part si vous voulez monter une société de nettoyage ${ }^{32}$ ".

La force de la fable réécrite par Pommerat réside bien sûr, comme tu le dis, dans ce renversement d'attitude de Sandra, qui d'esclave devient, non pas maître, mais l'égale d'un autre. Elle n'a pas besoin d'un prince charmant pour s'en sortir, mais d'une personne à qui parler, et avec qui partager l'expérience du deuil. D'ailleurs, leur relation- amour passager ou amitié durable? - n'est jamais clairement qualifiée ni établie, et la magnifique danse finale, qui les montre d'abord séparés, puis ensemble dans un rock and roll et un slow serré, puis de nouveau chacun de leur côté, dit à la fois leur autonomie et la force du lien qui les unit. L'épilogue insiste sur la persistance de leur relation par-delà la séparation. Ce pied-de-nez au traditionnel « Ils se marièrent et eurent beaucoup d'enfants ", est aussi une invitation à inventer d'autres modes de relations que ceux valorisés par les parents, obsédés par le lien conjugal (la belle-mère qui croit au prince charmant, le père qui veut refaire sa vie de couple à tout prix...).

BERENICE HAMIDI-KIM - Pour finir peut-être par là où ce dialogue avait commencé, je dirais que la différence entre Cendrillon et Ma Chambre Froide est double : parce que Pommerat assume cette fois de façon limpide de formuler une morale, et parce que la morale est ici aux antipodes du pessimisme anthropologique ${ }^{33}$ qui prévalait là-bas. Reste à savoir pourquoi : parce que c'est une adaptation d'un conte, une œuvre pour enfants... ou parce qu'elle ne s'attelle pas tant au monde social directement, comme dans les pièces qui donnent à voir le monde du travail, qu'à la trajectoire d'un sujet individuel envisagé dans ses relations familiales?

ARIANE MARTINEZ - Les trajectoires individuelles ne cantonnent pas pour autant la pièce dans des rapports universels et atemporels ; c'est aussi cela que j'aime en elle. Elle ne cesse de renvoyer, de manière allusive et décalée, parfois diluée et implicite, à la société actuelle. Les discours de la belle-mère, tout particulièrement, font écho à une idéologie de la fermeté, du dynamisme à tout crin, du volontarisme, idéologie qui était prégnante durant la Présidence de Nicolas Sarkozy. À la morale générale sur «les erreurs " qui " ont une fin », viennent ainsi s'adjoindre toute une série de morales parallèles: certaines sont effectivement atemporelles et s'inscrivent dans la 
perspective du conte (la nécessité pour chacun de mener son chemin de deuil); d'autres font plus nettement signe vers notre époque, comme par exemple la difficulté à départager les pragmatiques des rêveurs (la belle-mère qui affiche son ancrage dans le réel s'avère être la plus fantasque, celle qui s'est raconté le plus "d'histoires », et cette inversion de situation est aussi une leçon de lucidité). Cendrillon nous parle bien du monde dans lequel on vit.

\section{NOTES}

1. Bérénice Hamidi-Kim, «La Mauvaise âme de Ma Chambre froide», Frictions, $\mathrm{n}^{\circ} 19$, Paris, printemps-été 2012, p. 60-64.

2. «Il ne s'agit plus cependant de rechercher un usage social ou politique des textes, mais de questionner le besoin que nous avons de la figuration mentale de nos expériences pour les vivre. Sans accorder au théâtre le pouvoir de transformer le monde, nous le regardons comme un endroit où l'imaginaire peut à la fois se problématiser et se mettre au travait un « laboratoire de la conscience ", comme le formulait Stéphane [Braunshweig] à propos du théâtre de Kleist ; avec l'idée que mettre en mouvement nos représentations mentales transforme le champ de l'expérience et rouvre les possibilités de vivre- un tel postulat marque l'influence de la psychanalyse sur notre travail. » Anne-Françoise Benhamou, Dramaturgies de plateau, Besançon, Les Solitaires intempestifs, 2012, p. 101.

3. Bruno Bettelheim, La Psychanalyse des contes de fées, traduit de l'américain par Théo Carlier, Paris, Robert Laffont, 1976, p. 26.

4. Pour cette raison, ce personnage me parait jouer un rôle d'une importance cardinale, et assez conforme à l'original : un rôle de guide. Je ne suis donc pas sûre de partager l'avis de François Flahault quand il estime qu'elle " ne sert à rien ». N'aide-t-elle pas Cendrillon, même si elle est dépourvue de ses attributs merveilleux, ou plutôt précisément en ce qu'elle refuse de les utiliser parce qu'ils ne comblent pas un manque essentiel: celui du sens de l'existence, que seule la mortalité permet? Voir dans ce dossier, François Flahault, entretien avec Marion Boudier, «La rencontre de Cendrillon avec Blanche neige: décaler l'intrigue tout en respectant les enjeux fondamentaux du conte ".

5. Joël Pommerat, Cendrillon, Postface de Marion Boudier, Actes Sud, 2013, p. 53.

6. Joao Guimaraès Rosa, Diadorim, Paris, Albin Michel, 2006.

7. Donald Winnicott, La Mère suffisamment bonne, traduction Jeannine Kalmanovitch, Madeleine Michelin, Lynn Rosaz, Paris, Payot, 2006.

8. Françoise Davoine et Jean-Max Gaudilière, Histoire et trauma. La folie des guerres, Paris, Stock, 2004.

9. Voir le DVD Cendrillon de Joël Pommerat, réalisation Florent Trochel, livret d'accompagnement Marion Boudier, «Cendrillon, du spectacle au film », Axe Sud, 2014.

10. Alfredo Cañavate joue le père et le roi ; Noémie Carcaud joue la mère mourante, la grande soeur et la fée ; et Caroline Donnelly joue la petite sœur et le prince.

11. Joël Pommerat, dans Joëlle Gayot, Joël Pommerat, Troubles, Arles, Actes Sud, 2009, p. 48.

12. Christophe Triau, Cendrillon de Joël Pommerat, Sceren [CNDP-CRDP], Collection «Arts au singulier, THEATRE », 2013, p. 19-20. 
13. Bertolt Brecht, «Entretien sur les classiques [1929]», traductions par Jean-Louis Lebrave, Marielle Silhouette et Jean Tailleur, in Ecrits sur le théâtre, Collection "La Pléiade ", Paris Gallimard, 2000, p. 158-164. Dans cet entretien, Brecht refuse l'attitude de déférence à l'égard des classiques et défend l'idée qu'il faut « utiliser » les classiques et les " corriger » librement, comme la science a pu le faire avec les grandes découvertes du passé. Tout comme Brecht a pu réécrire des passages de Shakespeare dans La Résistible ascension d'Arturo Ui, et les transposer dans un «milieu prosaïque », Pommerat fait de même, avec Macbeth dans Cercles/fictions, ou avec La Mouette dans Cendrillon (lorsqu'il fait rejouer à la belle-mère la scène d'auto-comparaison entre Arkadina et Macha de l'acte II).

14. "Je n'écris pas des pièces, j'écris des spectacles, c'est comme ça. Je ne me suis pas dit : je vais écrire du théâtre. Je ne pense pas "texte". Le texte, c'est ce qui vient après, c'est ce qui reste après le théâtre. » Joël Pommerat, dans Joëlle Gayot, Joël Pommerat, troubles, Arles, Actes Sud, 2009, p. 19.

15. Joël Pommerat, Théâtres en présence, Arles, Actes Sud, 2007, p. 9.

16. Antonin Artaud, "La Mise en scène et la métaphysique " [conférence prononcée à la Sorbonne le 10 décembre 1931], in Le Théâtre et son double (1938), in CEuvres, Paris, Gallimard, 2004, Collection « Quarto Gallimard », p. 527.

17. Joël Pommerat, Théâtres en présence, op. cit., p. 17.

18. Ibid., p. 32.

19. Ibid., p. 31.

20. Antonin Artaud, « La Mise en scène et la métaphysique », op. cit., p. 527.

21. Joël Pommerat, dans Joëlle Gayot, Joël Pommerat, troubles, op. cit., p. 37.

22. Joël Pommerat, Cendrillon, op. cit., p. 112.

23. Je n'ai pas retrouvé cette phrase dans la version imprimée de la pièce, mais Deborah Rouach la prononce dans l'adaptation filmée (II, 12).

24. Joël Pommerat, Cendrillon, op. cit., p. 47.

25. Ibid., p. 21.

26. Ibid., p. 30.

27. «Elle fera sa petite vie avec sa mère... avec la robe de sa mère et voilà ! C'est pour nous que je fais ça en réalité ! Pour qu'on se retrouve nous ! Nous deux ! ", Ibid., p. 10.

28. Voir notamment :

L. D. , «La Reine des neiges, ou quand Disney avance d'un pas et recule de trois », Le Cinéma est politique, 23 décembre 2013, http://www.lecinemaestpolitique.fr/la-reine-des-neiges-ou-quanddisney-avance-dun-pas-et-recule-de-trois/ ;

Thomas Sotinel, "Chez Disney, la princesse a du mal à s'émanciper ", Le Monde, 19 novembre 2013, http://www.lemonde.fr/culture/article/2013/11/19/chez-disney-la-princesse-a-du-mal-as-emanciper_3516026_3246.html.

Pour un point de vue plus nuancé, voir Eddy Chevalier, « La Reine des neige de Disney est le film le plus sexuel de l'année ", blog rue 89, 05 décembre 2013, http://tempsreel.nouvelobs.com/ rue89/rue89-culture-pop/20131205.RUE9190/la-reine-des-neiges-de-disney-est-le-film-le-plussexuel-de-l-annee.html.

29. Joël Pommerat, Cendrillon, op. cit., p.46. La grande sœur, elle aussi, rappelle de manière fugitive le masochisme du conte initial, où les jeunes femmes se coupent les pieds pour le faire entrer dans le soulier de vair : « En tout cas, moi je me ferais couper un pied pour pouvoir voir le prince avant tous les autres » Ibid., p. 64.

30. Ibid., p. 60.

31. Simone Beauvoir, Le Deuxième sexe II, Partie 1, «Formation », Gallimard, Paris, 1976, Collection «Folio Essai », p. 123-124.

32. Joël Pommerat, Cendrillon, op. cit., p. 108. 
33. Sur le pessimisme anthropologique comme marque d'une certaine tendance du théâtre contemporain que j'ai appelé le «théâtre postpolitique », voir Bérénice Hamidi-Kim, Les Cités du théâtre politique en France depuis 1989, préface de Luc Boltanski, Montpellier, L'Entretemps, 2013.

INDEX

Mots-clés : Cendrillon, Pommerat (Joël), conte 